\title{
CARACTERIZACIÓN DE NIEVELES DE CONTAMINACIÓN EN UNA PLANTA EXTRACTORA DE AZUFRE UTILIZANDO IMÁGENES DE SATÉLITE DE ALTA RESOLUCIÓN PARA OPTIMIZAR EL PLAN DE MUESTREO
}

\author{
Martin H. BREMER BREMER*, Diego Fabián LOZANO GARCÍA, Mucio RODRÍGUEZ GARCÍA y \\ María del Consuelo HORI OCHOA
}

\author{
Instituto Tecnologico y de Estudios Superiores de Monterrey. Avenida Eugenio Garza Sada 2501, Colonia \\ Tecnológico Monterrey, Nuevo León, México, C.P. 64849 \\ *Autor para correspondencia: mbremer@itesm.mx
}

(Recibido enero 2015; aceptado octubre 2015)

Palabras clave: contaminación de suelo, evaluación ambiental, azufre, Jáltipan, Ikonos

\section{RESUMEN}

Las actividades industriales han generado un legado de impactos ambientales que representan un riesgo para los humanos. La sociedad está presionando a gobiernos e industriales para desarrollar trabajos de remediación de estos impactos, cuya documentación permite que sean empleados como ejemplo en tareas de limpieza de áreas contaminadas. Este estudio fue desarrollado en una mina de azufre abandonada en Jáltipan, Veracruz, que operó desde los años 50 hasta principios de los 90 , primero como una mina privada y después como dependencia del gobierno. Como era común en esa época, no había preocupación alguna sobre los impactos ambientales por la actividad de extracción, embalaje y envío de azufre. De ésta manera, de las 400 ha de la propiedad, aproximadamente el 20 \% quedó afectada por derrames de azufre. Este trabajo involucra dos aspectos: a) la determinación de las áreas contaminadas dentro y alrededor de la mina usando datos de alta resolución y SIG y b) la determinación de los volúmenes de material que requieren trabajos de remediación. Los resultados indican que el volumen a remediar dependerá de los objetivos de limpieza que impongan las autoridades ambientales correspondientes. Sin embargo, de acuerdo con los resultados es posible considerar como técnicamente viable la posibilidad de establecer como límite objetivo de remediación una acidez de $200 \mathrm{mg}$ $\mathrm{CaCO} 3 \mathrm{y} / \mathrm{o} \mathrm{pH} 4$. Esto implicará el remover las principales fuentes de contaminación: los depósito de carbón y azufre, los "vats" y los derrames de azufre. Dadas las condiciones del terreno, los procesos naturales permitirán neutralizar los suelos impactados y permitir el crecimiento de la cubierta vegetal.

Key words: soil pollution, site assessment, sulfur, Jaltipan, Ikonos

\begin{abstract}
Industrial activities in developing countries have left a legacy of environmental impacts that represent a long-term risk to human beings living in and around polluted areas. Fortunately, pressure from NGOs and social organizations is creating a trend of remediation projects that can be used as showcases in other areas, and stimulate both governments and private companies to initiate cleanup activities in polluted
\end{abstract}


areas all over the world. The study was conducted in a former sulfur mine in the city of Jaltipan, Veracruz, Mexico. This mine operated from the early 50s to early 90 s, first as a private company and later as a government company. As common in many mining companies during this period, there was virtually no concern for the environmental aspects of the mine operations, from mineral extraction to final product shipment. Thus, in an area of roughly 400 hectares, close to $20 \%$ of the area was affected by some kind of sulfur residues. This project involved two aspects. First, the determination of the polluted areas in and around the property of the mining company, using high-resolution imagery and GIS technologies in combination with extensive field surveys. Second, based upon the results of the diagnostic phase, the determination of the volume of polluted material, its three dimensional distribution and the selection of the best strategy for site remediation.

\section{INTRODUCCIÓN}

La extracción de recursos minerales puede impactar seriamente las condiciones ambientales del paisaje, alterando patrones de drenaje, de uso y cobertura del suelo, así como contaminando el suelo y agua de las áreas en donde se desarrollan estas actividades. El uso de técnicas de percepción remota (PR) y sistemas de información geográfica (SIG) en combinación con muestreo, análisis químicos de compuestos y otras técnicas geoambientales han sido muy útiles para identificar y cuantificar los impactos creados por la actividad humana sobre la superficie terrestre. Las actividades mineras pueden impactar de manera negativa sobre los ecosistemas, haciendo que sea importante el estimar dichos impactos y proponer medidas de mitigación o remediación, acorde con las condiciones locales.

Las imágenes satelitales se han usado como un complemento para realizar inventarios de las zonas contaminadas en aquellos lugares en donde se carece de una información fidedigna sobre la extensión de las áreas afectadas. En este contexto, en ocasiones, cuando los contaminantes tienen respuestas evidentes en las ventanas espectrales que manejan los sensores remotos, las técnicas de PR permiten obtener resultados más confiables que otras herramientas (particularmente trabajo de campo) y facilitan la ubicación geográfica de los lugares contaminados, lo que hace más sencillo su incorporación a los SIG para su posterior análisis.

Los datos de PR incorporados a un SIG representan una poderosa herramienta para el manejo, estudio y modelación de áreas contaminadas. Asimismo, facilitan la obtención de mapas de los niveles de contaminación por actividades mineras de las zonas que deben ser restituidas para permitir la ocupación humana o la restauración de los ecosistemas anteriormente presentes en el área.
Los avances científicos en los métodos de análisis y de integración de datos o información representan un progreso de indudable interés hoy en día en el ámbito de las geociencias. Los SIG brindan un apoyo tecnológico que facilita el desarrollo, cada vez más extendido, de este tipo de aplicaciones en las que en esencia se analiza información multitemática georreferenciada (Cassard et al. 2008, Chica-Olmo et al. 1994, 2002 y 2008, Carranza 2009).

Los datos del tipo Landsat-TM y SPOT han sido empleados por varios autores para el monitoreo de áreas impactas (Mularz 1998, Rigol y Chica-Olmo 1998, Kettles 2000). Con la disponibilidad de datos de alta resolución espacial del tipo Ikonos o Quickbird, se facilita la identificación de pequeñas zonas impactadas, que al sumarse pueden llegar a representar un porcentaje importante del área afectada y por tanto un volumen considerable para su remediación.

Rigol-Sánchez et al. (2011) aplicaron modelos de integración de datos espaciales por los métodos de suma ponderada multiclase y el de regresión logística múltiple, para depósitos metálicos en el sureste de España, donde los SIG han generado mapas predictivos, por medio del cálculo de un índice de favorabilidad minera (IFM), que ha facilitado la selección de las zonas con mayor potencial para albergar depósitos minerales. Los resultados obtenidos indican que el rendimiento de los modelos es similar en muchos de los experimentos, con porcentajes de acierto de depósitos conocidos sólo un poco superiores en los modelos basados en regresión logística. No obstante, el método de suma ponderada multiclase puede ser aceptable en la mayoría de los casos.

Las zonas contaminadas de la explotación minera bajo estudio (actualmente fuera de producción) son reconocibles en una imagen de resolución media y tienen suficientes diferencias espectrales respecto 
a los otros tipos de suelo de los alrededores como para permitir la representación de su distribución mediante técnicas de clasificación supervisada y no supervisada. Las respuestas de reflectancia espectral de los residuos son suficientemente diferentes para permitir la separación respecto a los otros usos y coberturas del suelo de las regiones adyacentes usando imaginería Landsat-TM. Las zonas contaminadas son muy reconocibles en las bandas 7-4-2 de las imágenes Landsat y también en las ortofotos usadas en el análisis de la precisión de los resultados (Joan 2005).

Las imágenes del satélite Landsat 5, con las que trabajaron Rebordosa y López (2009), tienen una resolución espacial insuficiente para acometer decisiones en el día a día de una actividad extractiva. Estos autores afirman que existen satélites civiles de observación de la Tierra con resoluciones espaciales de $0.5 \mathrm{~m}$ para sensores pancromáticos y de $2.5 \mathrm{~m}$ para sensores multiespectrales. El costo de sus productos, a pesar de ser muy superior al de las imágenes Landsat, irá a la baja y en todos los casos la relación precio/hectárea observada es muy inferior a la de la fotografía aérea, sensores hiperespectrales a bordo de avión, o el mismo trabajo de campo.

La clasificación digital de imágenes de satélite se ha convertido en una herramienta indispensable para monitorear la cobertura terrestre. Debido a los errores que se observan a menudo en los mapas derivados del análisis de datos de percepción remota, existe una búsqueda constante por desarrollar y probar alternativas que permitan la obtención de resultados precisos para la toma de decisiones. García Mora y Mas (2008) trabajaron con una imagen Landsat en el sureste de México y compararon una clasificación tradicional (desarrollando el análisis pixel por pixel con las firmas espectrales) con otros procedimientos de clasificación de la imagen (contextual, por redes neuronales y añadiendo datos auxiliares). Los resultados obtenidos muestran que la incorporación de datos auxiliares representados por imágenes de probabilidad a priori permite aumentar hasta un $20 \%$ la fiabilidad en comparación con una clasificación convencional, superando incluso los resultados obtenidos con métodos no paramétricos y contextuales. Sin usar la información a priori, los tres métodos de clasificación (máxima verosimilitud, clasificador de contexto SMAP y perceptor multicapa) dan resultados cuya fiabilidad es parecida, la prueba de McNemar no encontró diferencia significativa entre ellos. La fiabilidad alcanzada por los dos métodos para los cuales se utilizó la información a priori (máxima verosimilitud y perceptor multicapa) es similar, no se encontró una diferencia estadísticamente significativa. El único método que presentó una mejora significativa en la clasificación es la incorporación de datos adicionales y no el algoritmo de clasificación. La utilización de información adicional a la información espectral a través del uso de probabilidades a priori es por lo tanto una alternativa promisoria para mejorar el mapeo y monitoreo de las coberturas del suelo con base en datos de percepción remota.

En la actualidad, se aplican con éxito tecnologías muy eficaces como la teledetección y los SIG para identificar la huella espacial de impactos y apoyar las decisiones que ayuden a tomar medidas correctivas y prospectivas. Ponvert-Delisles y Lau-Quan (2013), aplicaron las tecnologías de teledetección y los SIG en la evaluación de impactos provocados por el empleo de equipamiento y tecnologías del campo de la ingeniería agrícola. Donde se obtuvo un cartograma de salinidad y fertilidad de los suelos con imágenes de satélite de muy alta resolución espacial (Ikonos) asociados con variables ambientales y físicas. Además, se usaron diversos algoritmos de clasificación que se apoyan en variables detectadas por satélites, mediciones de campo y análisis con SIG para la zonificación del fenómeno.

La teledetección hiperespectral aerotransportada puede ser utilizada para detectar hidrocarburos eficientemente, ya que los hidrocarburos se caracterizan por absorción en los espectros. La disponibilidad de datos Hymap (cuya razón señal-ruido es muy alta) permite la clasificación de pixeles que indiquen contaminación con hidrocarburos, inclusive a niveles de concentración bajos. Los suelos contaminados por aceites u otras substancias similares pueden ser identificados directamente en la imagen sin ambigüedad y sin requerir un proceso previo de corrección atmosférica (Hörig 2001).

\section{Azufrera Panamericana}

La ex-Azufrera Panamericana es una mina de azufre abandonada, localizada en las afueras de la ciudad de Jáltipan, Veracruz (Fig. 1). Al inicio del siglo XX, los trabajos de exploración de petróleo y gas en esta región localizaron un domo salino que tenía un depósito de azufre asociado a la presencia de sal y petróleo en el subsuelo. La producción de azufre en la mina se inició a mediados del siglo pasado y continuó hasta principios de los años 90 .

El azufre era extraído por medio del método Frasch. Con este sistema se perforan pozos hasta el depósito de azufre, al que se le inyecta vapor de agua que lo licúa y finalmente es extraído a la superficie 


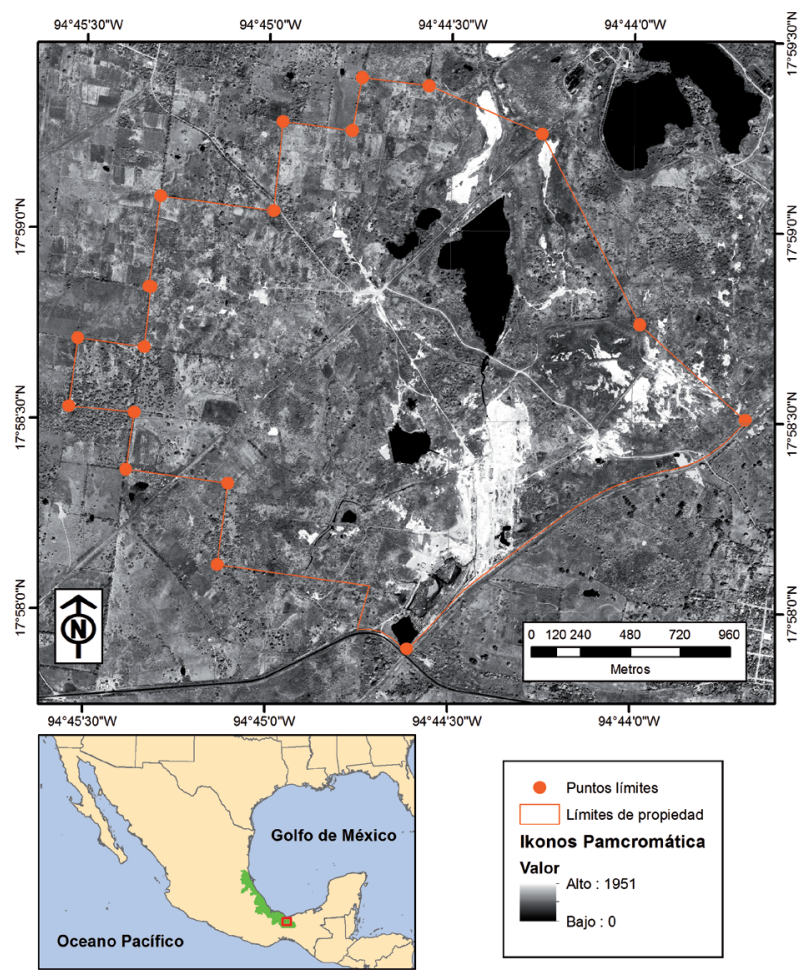

Fig. 1. Ubicación del área de estudio, con puntos que indican los límites de propiedad

por medio de bombas de presión. El mineral de azufre se separa del agua por evaporación. Este proceso realizado a cielo abierto en las áreas de almacenaje para transporte llamadas "vats", tuvo un fuerte impacto sobre los suelos y cuerpos de agua de la zona por los derrames de la mezcla agua/azufre y la producción de agua con altos niveles de acidez. El azufre fundido, al ser muy corrosivo, provocó constantemente rupturas de las tuberías que lo transportaban de los pozos a la planta de ácido y a los vats, lo que ocasionó múltiples derrames a lo largo y ancho del predio que no fueron atendidos en su momento y actualmente también representan fuentes de aguas ácidas. Además, se empleaba carbón en el proceso de purificación, lo que generó un residuo que era depositado en un área específica del terreno de la mina y que representa (incluso actualmente) un peligro, ya que es un material de fácil combustión que ha ocasionado incendios espontáneos, junto con la emisión de gases tóxicos que ha derivado en la evacuación de los pobladores en la cercanía de Jáltipan.

Los objetivos del presente trabajo fueron localizar y delimitar las áreas impactadas por las actividades mineras por medio de métodos multidisciplinarios de muestreo, análisis químicos, SIG y PR de una forma efectiva en función de los costos, dada la extensión y cobertura vegetal del terreno estudiado que hacían muy difícil realizar una campaña confiable utilizando únicamente estrategias tradicionales de muestreo. Para alcanzar los objetivos anteriores se plantearon los siguientes objetivos particulares: diseñar un plan de muestreo que identificara adecuadamente las condiciones de contaminación del predio en cuestión, identificar y cuantificar las áreas afectadas por derrames de azufre y otros contaminantes con imágenes de alta resolución (Ikonos), representar la extensión lateral de las áreas contaminadas en un SIG para su posterior modelación espacial, calcular los volúmenes de material contaminado y plantear estrategias de remediación con base en los resultados de la evaluación.

\section{MATERIALES Y MÉTODOS}

El estudio abarcó un área de 480 ha (Fig. 1), las cuales fueron divididas en las siguientes zonas: a) depósito de carbón y azufre, b) depósitos de azufre y vats, c) derrames de azufre ocasionados a lo largo de ductos y otras instalaciones, d) presas y lagunas de acumulación de los escurrimientos pluviales, e) pastizales y zonas de cultivo y f) vegetación secundaria afectada por actividades humanas no relacionadas con la mina.

\section{Puntos de control y límites de propiedad}

Se emplearon dos GPS Magellan ProMark XCM con antena hemisférica para ubicar un punto geodésico de Instituto Nacional de Estadística y Geografía (INEGI) y propagar las coordenadas para tener un punto de referencia base dentro de los terrenos de la mina. Se identificaron y levantaron coordenadas en campo ya que los límites de la propiedad no estaban georeferenciados. Se obtuvo un total de 30 puntos correspondientes a los vértices de los límites de la propiedad (Fig. 1) por medio de un procedimiento diferencial.

\section{Imagen de satélite Ikonos}

La imagen de satélite Ikonos (multiespectral y pancromática) se georreferenció empleando puntos de control GPS identificados en campo. La figura 2 muestra la imagen multiespectral con la ubicación de varios rasgos importantes dentro y fuera de la propiedad de la mina. Los derrames de azufre y los suelos desnudos se muestran de color cian (\# 1 y \# 2 en la imagen), el depósito de carbón y azufre aparece con tonos grises (\#3), mientras que las lagunas aparecen en colores azul oscuro-negro (\# 4 y \# 5).

Los datos de PR fueron de gran utilidad para identificar las zonas afectadas por derrames de azufre. 


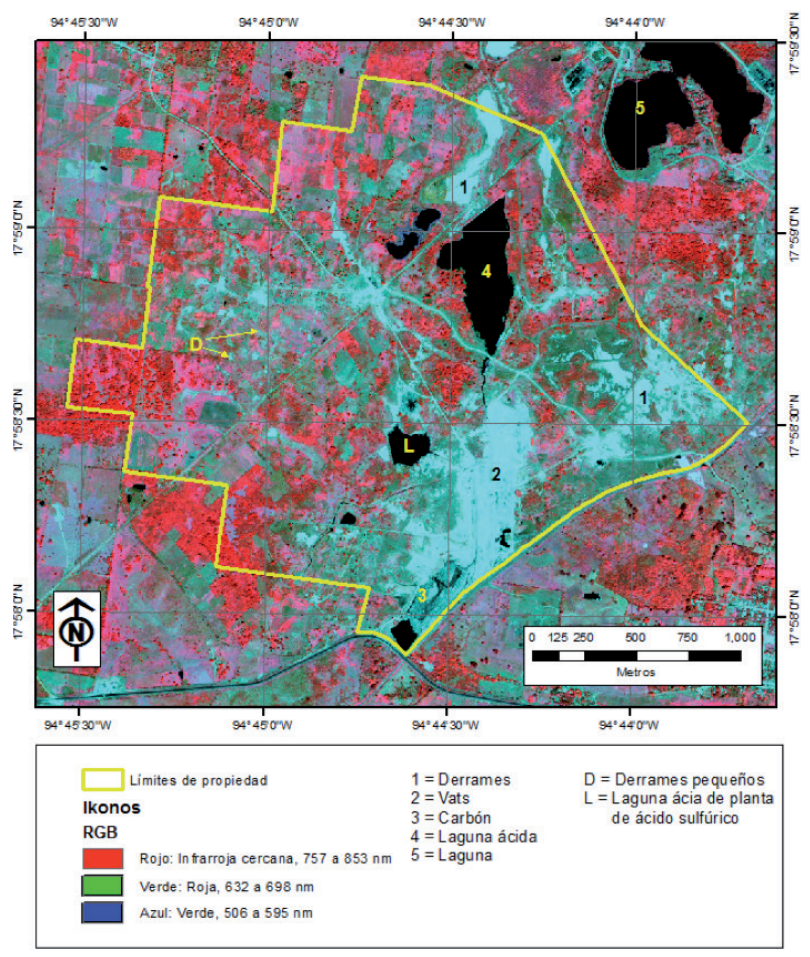

Fig. 2. Imagen multiespectral Ikonos. Derrames de azufre (1), área de vats (2), depósito de carbón y azufre (3), lagunas (4 y 5)

En particular, aquellos derrames que cubrían una superficie pequeña y que eran difíciles de identificar en campo por la altura de la vegetación. La información generada con la imagen se complementó con datos de campo e información existente del domo salino y la extracción de azufre.

Adicionalmente, se emplearon fotografías aéreas estereoscópicas para obtener un modelo tridimensional del terreno e interpretar el patrón de drenaje y la dirección del flujo de agua superficial de forma visual y con la ayuda de trabajo de campo.

\section{Muestreo de campo}

Inicialmente el muestreo fue diseñado a partir de los recorridos de campo. Se planteó obtener dos puntos de muestreo por hectárea como base para determinar tendencias regionales de contaminación y se consideró utilizar patrones de muestreo a mayor densidad en zonas donde hubiera derrames y restos de azufre como en el caso de los vats, el depósito de carbón y azufre, así como los derrames. El plan de muestreo se diseñó bajo el sistema de muestreo estratificado según las condiciones esperadas de contaminación. Las clases de muestreo propuestas fueron: agua y sedimentos de presas, derrames, vats, depósito de carbón y azufre y muestreo regional (suelos). El muestreo de campo se dividió según los tipos, densidades de muestras y profundidades de muestreo, a partir de las condiciones esperadas de contaminación presentes.

En cuanto se obtuvo la imagen satelital de alta resolución, adicionalmente a la malla regular de puntos definida con anterioridad, se añadieron nuevos sitios de muestreo ya que se identificaron más derrames potenciales en la imagen multiespectral debido a que durante el recorrido de campo se subestimó la cantidad de derrames y otros sitios contaminados en el terreno. Esto implicó replantear la densidad de puntos regionales y tener margen para el incremento substancial de muestras a realizar en dichos derrames sin cambiar el número presupuestado del total de muestras.

La figura 3 presenta la ubicación de los puntos de muestreo realizados dentro de los límites de la propiedad, en la que se muestrearon a nivel superficial todos los puntos regionales. Además, se tomaron muestras a intervalos de $50 \mathrm{~cm}$ de profundidad en los puntos localizados en los vats y los derrames. Los muestreos en el depósito de carbón y azufre requirieron de una perforadora mecánica para obtener muestras hasta $10 \mathrm{~m}$. Para los puntos ubicados en las lagunas se obtuvieron muestras de agua y sedimentos.

La profundidad inicial de extracción de todas las muestras fue de $30 \mathrm{~cm}$. Para aquellos sitios con

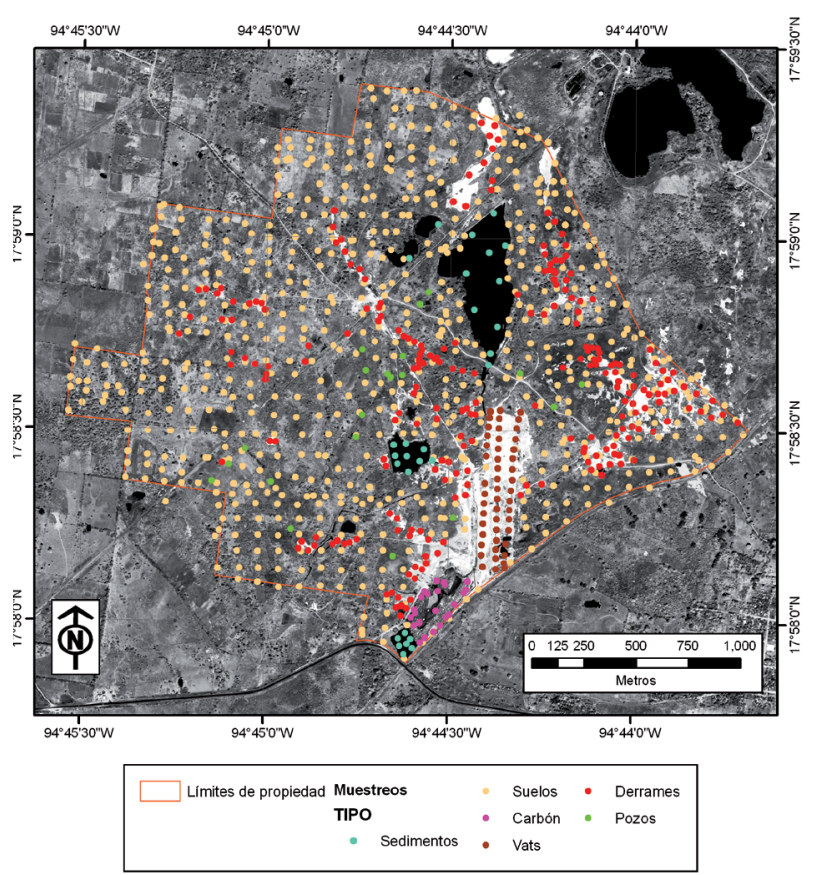

Fig. 3. Puntos de muestreo en la ciudad de Jáltipan, Veracruz, México 
niveles de contaminación alta (depósito de carbón y azufre, vats y derrames), se extrajeron muestras cada $50 \mathrm{~cm}$ de profundidad, hasta que por inspección visual se observaba que no había contaminación.

\section{Análisis de laboratorio}

Todas las muestras fueron procesadas en los Laboratorios Analíticos del Centro de Calidad Ambiental del Instituto Tecnológico de Monterrey (ITESM). Se construyó una base de datos con los resultados de los análisis (acidez y pH), registrando además los datos de localización $(\mathrm{X}-\mathrm{Y})$, profundidad y tipo de muestreo.

La determinación de la acidez intercambiable se basa en el uso de una sal neutra como el cloruro de potasio $(\mathrm{KCl})$ con un ion desplazante $(\mathrm{K}+)$, el cual provoca que los iones ácidos (aluminio $(\mathrm{Al}+$ ) e hidronios $(\mathrm{H}+))$ pasen a la solución. Esta acidez es luego titulada con una solución básica, de modo que la cantidad de acidez será igual a la cantidad de base usada entre los puntos de neutralización con fenolftaleína. $\mathrm{El} \mathrm{pH}$ fue medido en laboratorio con el método analítico NMX-AA-008-1980.

\section{Modelación espacial}

Los datos resultantes de los muestreos y análisis químicos fueron procesados para generar dos nuevas capas de información con los valores de $\mathrm{pH}$ y acidez. Se realizaron interpolaciones en dos dimensiones usando dos algoritmos diferentes: inverso del cuadrado de las distancias ponderadas (IDW, por sus siglas en inglés) para los datos de acidez y kriging ordinario para los datos de $\mathrm{pH}$.

\section{RESULTADOS}

La imagen Ikonos fue modificada para realzar las áreas afectadas por las actividades mineras y visualizar con mayor resulución las áreas con contaminación por azufre y así, diferenciarlas de los suelos desnudos (Fig. 2). Lo anterior permitió replantear el plan original de muestreo y planear una distribución más efectiva de los trabajos de campo para colecta de muestras de suelo y su posterior análisis en el laboratorio.

La figura 4 presenta el área de vats ampliada y realzada para mostrar las diferencias espectrales producidas por las condiciones diversas de contaminación de azufre, mezcla de suelos y presencia de agua en el suelo. En el recuadro izquierdo de la figura 4, se muestra la imagen en falso color con el histograma calculado a partir de todo el conjunto de datos.

En la imagen de la derecha de la misma figura aparece la zona de vats ampliada con el histograma calculado sobre esa área únicamente. Se pueden observar las diferencias de tonalidad producidas por distintos niveles de contaminación de azufre (tonos amarillos y rosas), las áreas con suelo y azufre (tonos grises), así como las áreas con agua (tonos negros). Esta imagen permitió diseñar el muestreo en la zona de vats para tener una mejor idea de la distribución horizontal y vertical de la contaminación por azufre.

\section{Clasificación de la imagen Ikonos}

La imagen Ikonos se clasificó para identificar los tipos básicos de cobertura del suelo (Fig. 5). Se empleó un método híbrido para generar las estadísticas de

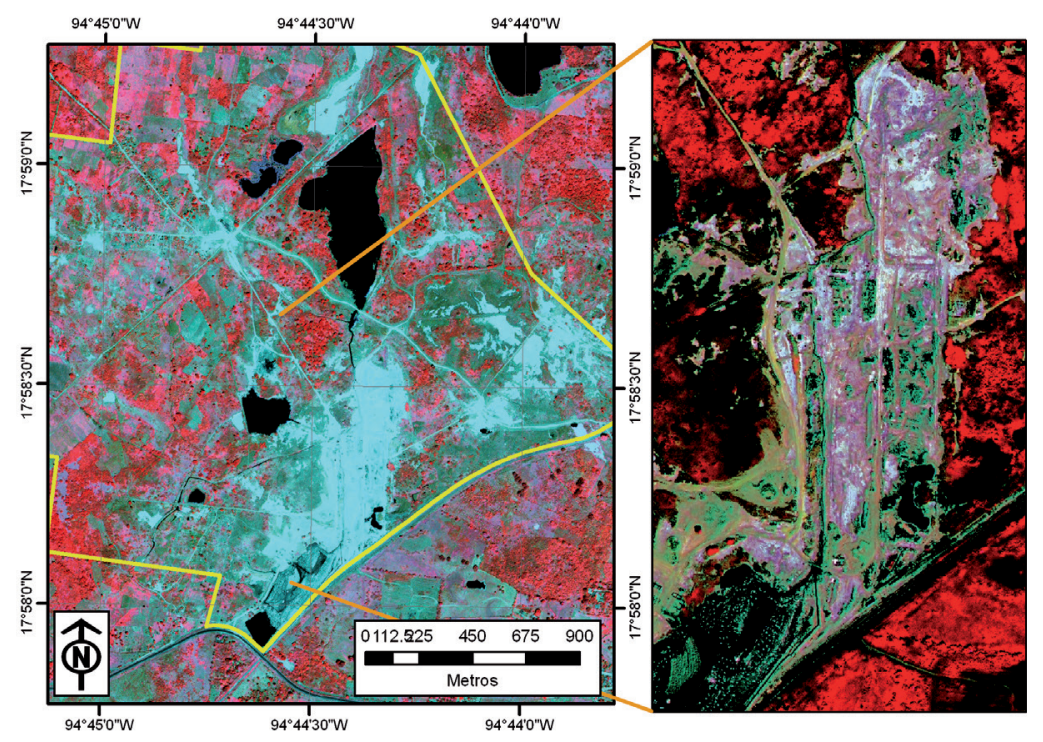

Fig. 4. Realce de imagen Ikonos en la zona de vats 
entrenamiento (supervisadas y no supervisadas), posteriormente se realizó un análisis de grado de separación de las estadísticas resultantes por medio de un algoritmo de divergencia transformada, y finalmente se clasificó la imagen usando un clasificador de máxima verosimilitud. Las clases identificadas se muestran en la figura 4 y el área cubierta por cada clase aparece en la cuadro I.

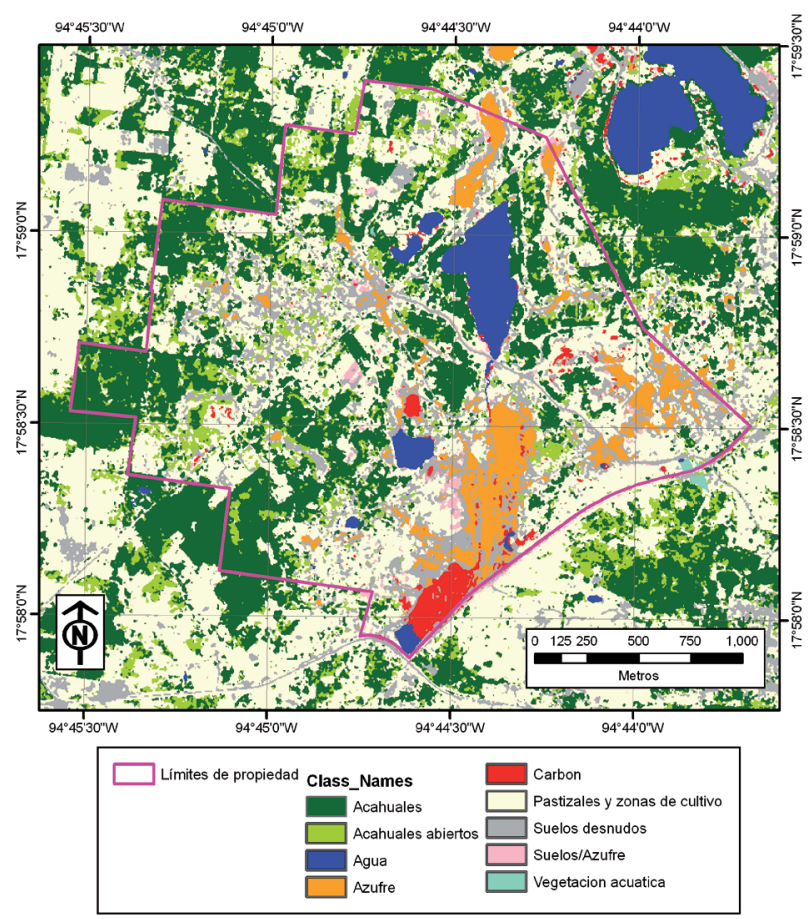

Fig. 5. Clasificación de la cobertura del suelo de la imagen Ikonos

\section{Patrones de drenaje hidrológico}

La figura 6 muestra el resultado final de la cartografía de la red hidrológica. La información permitió identificar la dirección básica del flujo de agua para posteriormente definir el sitio más adecuado para almacenar los residuos de la remediación del sitio. Se observan dos direcciones principales en el flujo de agua. La primera es hacia el norte, en dirección al río Chacalapa (fuera de la zona de estudio) y la segunda hacia el sur, cruzando la carretera México. 180 a través de un ducto localizado a $650 \mathrm{~m}$ al oeste del acceso principal de la mina.

\section{Muestreos}

Se obtuvieron un total de 1400 muestras en 1076 puntos para los distintos tipos de condiciones identificadas en campo (Cuadro II). El diseño inicial (previo a la obtención de la imagen satelital) contempló abarcar un punto por hectárea. Una vez
CUADRO I. CLASIFICACIÓN DE IMAGEN IKONOS

\begin{tabular}{lcc}
\hline Cobertura & Hectáreas & $\%$ \\
\hline $\begin{array}{l}\text { Cuerpos de agua: Lagunas, áreas de } \\
\text { almacenamiento de agua, ríos y riachuelos. }\end{array}$ & 20.75 & 4.2 \\
\hline $\begin{array}{l}\text { Vegetación secundaria: Áreas de cultivo } \\
\text { abandonadas en distintos estados de } \\
\text { recuperación (Acahuales). }\end{array}$ & 182.13 & 37.0 \\
\hline $\begin{array}{l}\text { Pastizales y cultivos: Áreas bajo uso } \\
\text { agrícola, ya sea pastizales para ganado o }\end{array}$ & 158.45 & 32.2 \\
$\begin{array}{l}\text { zonas de cultivo. } \\
\begin{array}{l}\text { Vegetación acuática: Vegetación asociada } \\
\text { a cuerpos de agua. }\end{array}\end{array}$ & 0.40 & 0.1 \\
\hline $\begin{array}{l}\text { Suelos desnudos: Áreas sin vegetación } \\
\text { aparente, caminos, zonas de cultivo sin } \\
\text { vegetación. }\end{array}$ & 73.99 & 15.1 \\
\hline
\end{tabular}

Zonas incendiadas: Áreas agrícolas que fueron incendiadas en la época de toma de la imagen.

\begin{tabular}{lcc}
\hline Depósito de carbón y azufre. & 13.18 & 2.7 \\
\hline $\begin{array}{l}\text { Depósitos de azufre: Áreas con derrames } \\
\text { de azufre. }\end{array}$ & 33.04 & 6.7 \\
\hline $\begin{array}{l}\text { Suelos/Azufre: Áreas con mezcla de } \\
\text { suelos y azufre. }\end{array}$ & 9.58 & 1.9 \\
\hline
\end{tabular}

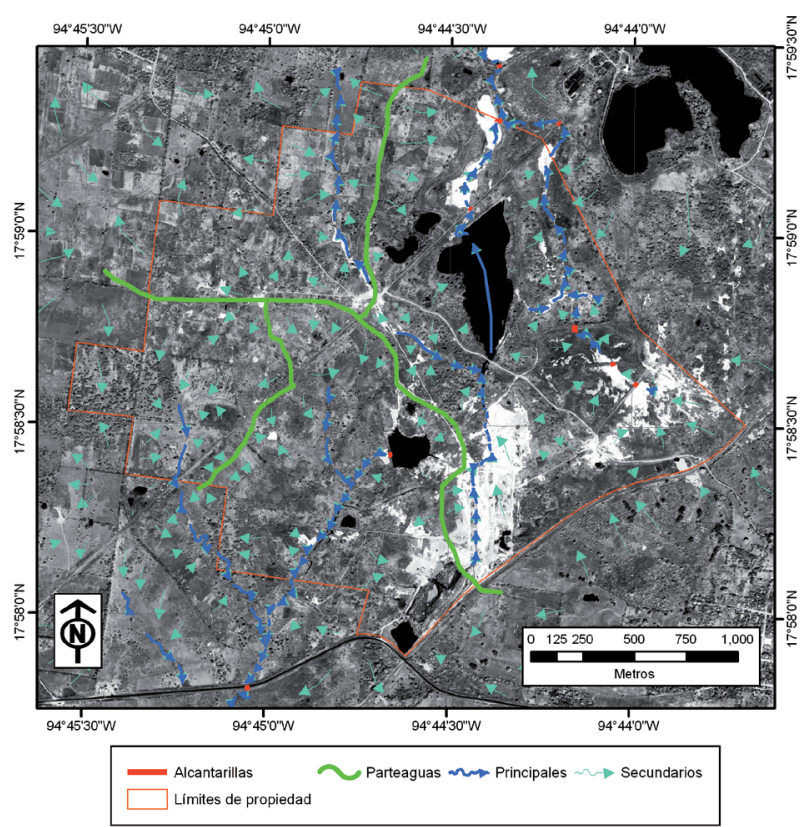

Fig. 6. Patrones de drenaje

que se tuvo la imagen se agregaron puntos de muestreo correspondientes a sitios identificados como "derrames", "pozos" y “carbón” (Fig. 3, Cuadro II). 
Los rangos de acidez y de $\mathrm{pH}$ varían de 0.398 a 800 y de 1.2 a 10.0, respectivamente (Cuadro III).

CUADRO II. TIPO DE MUESTREOS REALIZADOS EN LA ZONA DE ESTUDIO

\begin{tabular}{lcc}
\hline Tipo & \# puntos & \# muestras \\
\hline Sedimentos (lagunas) & 28 & 28 \\
Suelos & 663 & 693 \\
Carbón & 23 & 75 \\
Vats & 49 & 115 \\
Derrames & 272 & 448 \\
Pozos & 41 & 41 \\
Total & 1076 & 1400 \\
\hline
\end{tabular}

\section{Cartografía de la contaminación}

Se desarrollaron los planos de acidez y de $\mathrm{pH}$ a partir de una interpolación de los resultados del muestreo. Los mapas se generaron con dos algoritmos diferentes. El mapa de pH (Fig. 7) se generó con un algoritmo de kriging, mientras que el mapa de acidez se generó con un algoritmo de IDW (Fig. 8). Los resultados de la estimación del error (para el caso del $\mathrm{pH}$ ) empleando kriging ordinario, se muestran en la figura 9.

En el cuadro IV se muestran los resultados de las estadísticas descriptivas de los datos medidos y de los interpolados. En las figuras 10 y 11 se muestra el resultado de la correlación entre los datos medidos en campo y laboratorio y los interpolados, así como el intervalo de confianza de 95 $\%$ de dicho resultado.

\section{DISCUSIÓN}

La ubicación de los puntos de muestreo de la malla regular fue definida con base en las primeras visitas de campo. En salidas posteriores fue evidente que se requería contar con una herramienta que permitiera



Fig. 7. Valores de $\mathrm{pH}$ muestreados e interpolados

visualizar la totalidad de la zona de estudio debido a que la cobertura vegetal presente en ésta (principalmente acahuales de vegetación secundaria con crecimiento de entre 3 y $4 \mathrm{~m}$ de altura), impedía la observación de características y rasgos en el terreno desde una perspectiva terrestre.

Aunque en una etapa temprana del proyecto se tuvo acceso a fotos aéreas del sitio, éstas no eran recientes y sólo se podían distinguir tonalidades de gris. Por ello no era posible observar en esas fotos algunos rasgos presentes en el terreno, razón por la cual se decidió adquirir una imagen satelital de alta resolución cuya fecha de toma correspondiera al inicio del proyecto.

CUADRO III. RANGOS DE ACIDEZ Y pH PARA LOS DISTINTOS TIPOS DE MUESTREO

\begin{tabular}{lccccc}
\hline \multirow{2}{*}{ Tipo } & \multicolumn{2}{c}{ Acidez $(\mathrm{cmol} / \mathrm{kg})$} & & \multicolumn{3}{c}{$\mathrm{pH}$} \\
\cline { 2 - 3 } \cline { 5 - 6 } & mínimo & máximo & & mínimo & máximo \\
\hline Sedimentos (lagunas) & 12.56 & 453.33 & & 2.6 & 7.4 \\
Suelos (muestreo regional) & 1.69 & 246.30 & & 2.72 & 15.72 \\
Carbón & 7.30 & 541.18 & & 1.36 & 7.44 \\
Vats & 9.76 & 800.48 & & 1.03 & 8.0 \\
Derrames & 2.94 & 1339.89 & & 1.37 & 8.72 \\
Pozos & 13.72 & 50.30 & & 7.70 & 9.66 \\
\hline
\end{tabular}




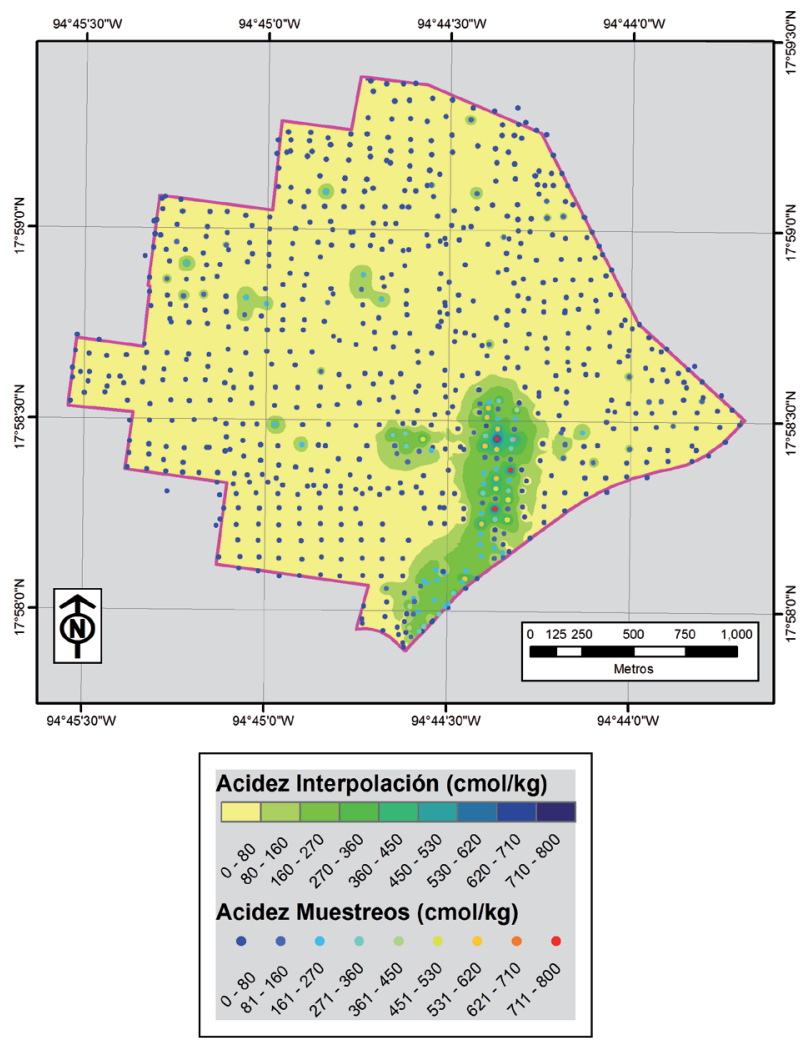

Fig. 8. Valores de acidez muestreados e interpolados

De esta manera, uno de los elementos más útiles en este estudio fue el uso de una imagen satelital multiespectral de alta resolución con fecha reciente. Con esta imagen se logró visualizar las condiciones del predio prácticamente en el momento en que se realizaba el trabajo de campo.

Los puntos de muestreo, reclasificados posteriormente al plan inicial de muestreo, obedecieron a la identificación de la posible presencia de una mayor cantidad de derrames, interpretados a partir de la imagen satelital. Muchos de estos sitios probablemente no hubieran podido ser localizados, ya que su tamaño era pequeño. Ejemplos de estos sitios se señalan en la figura 2, marcados con la letra " $\mathrm{D}$ ".

Para el cálculo del volumen a remediar es necesario determinar el grado de acidez que será

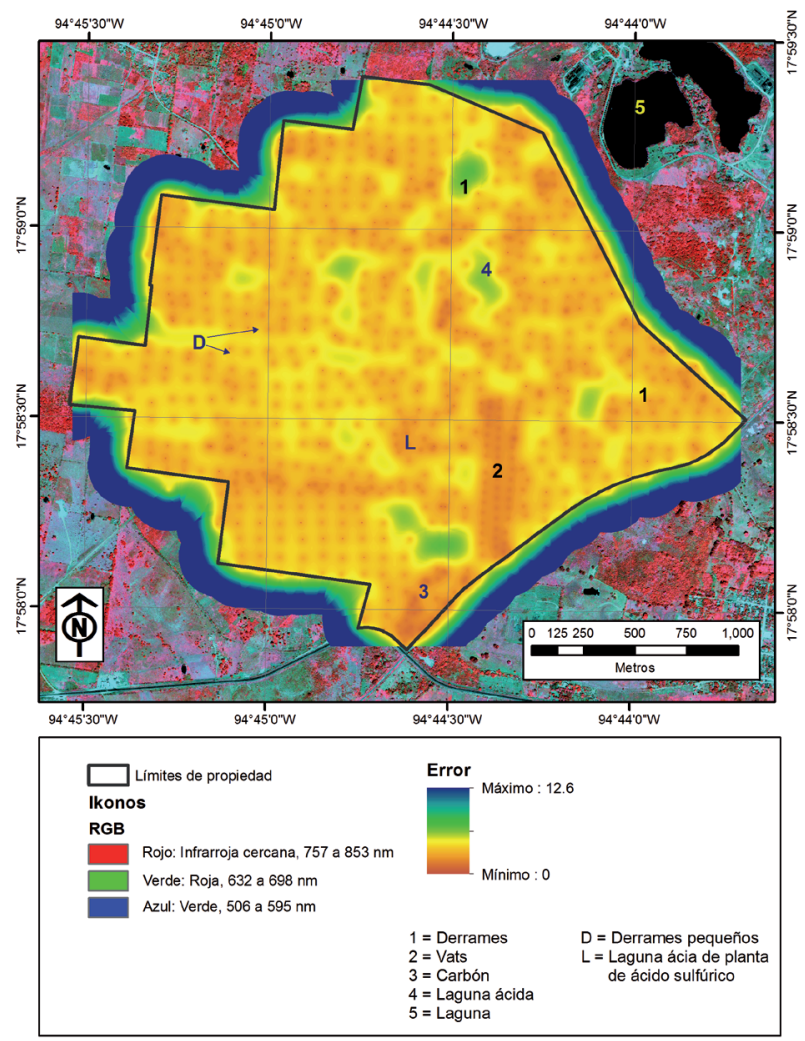

Fig. 9. Mapa de la predicción del error en la estimación de pH por el método de kriging

considerado como máximo permitido. Debido a que en la fecha del estudio no había normatividad de acidez máxima en suelos, se realizaron cálculos de volumetría con dos diferentes niveles de acidez para identificar el porcentaje de aumento del volumen a remediar utilizando un límite más estricto (Cuadro V).

La zona de estudio drena hacia dos cuencas. En la porción norte y este, los escurrimientos llegan al río Chacalapa. Estos escurrimientos drenan principalmente la zona de vats, que era la zona de carga al ferrocarril para la venta del azufre. Esta zona presentaba una gran cantidad de azufre en la superficie y se llegaron a encontrar trozos de azufre elemental de considerable tamaño. El cuerpo receptor del drenaje era una laguna artificial ácida (la de mayor

CUADRO IV.ESTADÍSTICAS DESCRIPTIVAS DE LOS RESULTADOS DE MEDICIÓN EN LABORATORIO E INTERPOLACIÓN PARA LOS PARÁMETROS DE ACIDEZ Y pH

\begin{tabular}{lccccc}
\hline Variables & Observaciones & Error cuadrático medio & $\mathrm{R}^{2}$ & $\mathrm{R}^{2}$ ajustada & RECM \\
\hline ACIDEZ & 766 & 123.791 & 0.989 & 0.989 & 11.126 \\
$\mathrm{pH}$ & 766 & 0.064 & 0.983 & 0.983 & 0.253 \\
\hline
\end{tabular}

$\mathrm{R}^{2}=$ raíz cuadrada, $\mathrm{RECM}=$ raíz cuadrada del error cuadrático medio 


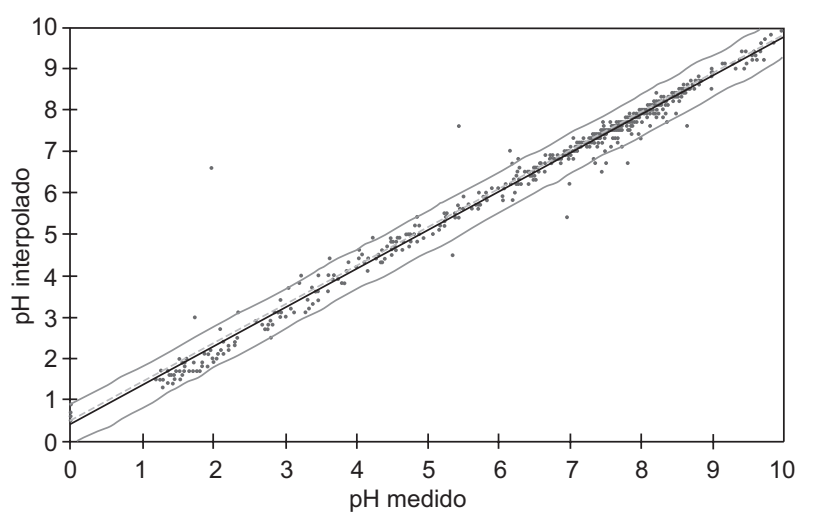

$\left(R^{2}=0.983\right)$

\begin{tabular}{|c|c|}
\hline $\begin{array}{|ll|}- & \text { Datos } \\
& \text { Int. Conf. (media 95\%) }\end{array}$ & $\begin{array}{l}- \text { Modelo } \\
\text { Int. Conf. (Obs. 95\%) }\end{array}$ \\
\hline
\end{tabular}

Fig. 10. Valores de $\mathrm{pH}$ medidos $v s$. interpolados. Int. Conf. = Intervalo de confianza, Obs. $=$ Observado

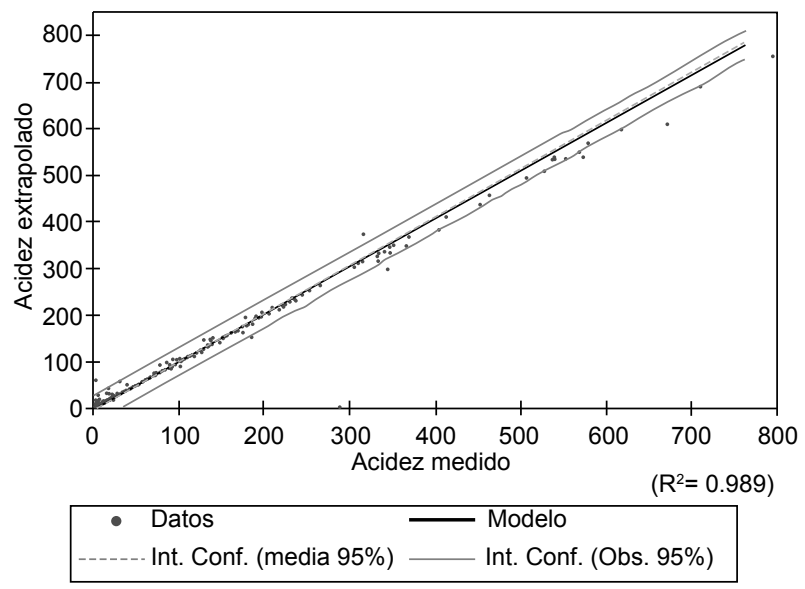

Fig. 11. Valores de acidez medidos vs. interpolados. Int. Conf. $=$ Intervalo de confianza, Obs. $=$ Observado

superficie dentro de los límites de la propiedad), la cual fue construida para minimizar la aportación de agua con un bajo nivel de $\mathrm{pH}$ al río.

La otra dirección principal de drenaje tiene orientación hacia el sur y drena dos elementos importantes dentro de la propiedad: el depósito de carbón y azufre y la laguna de la planta de ácido sulfúrico (Fig. 2, L). Estas zonas también presentaban valores de $\mathrm{pH}$ muy bajos y representaban un serio peligro aguas abajo de la mina.

Los resultados de la clasificación de la imagen Ikonos muestran las condiciones del terreno durante el trabajo de campo. Además del uso de la imagen para identificar más sitios de muestreo, los datos de cobertura del suelo permitieron ubicar los límites de las áreas contaminadas, lo cual fue de gran ayuda para
CUADRO V. VOLÚMENES DE SUELO $\left(\mathrm{m}^{3}\right)$ A REMEDIAR SEGÚN EL LÍMITE DE ACIDEZ (cmol/ $\mathrm{kg}$ ) QUE SEA PROPUESTO POR LA AUTORIDAD

\begin{tabular}{lcc}
\hline Área & Acidez $>100$ & Acidez $>200$ \\
\hline Depósito de carbón y azufre & 309593.49 & 309593.49 \\
Vats & 273024.60 & 272234.89 \\
Derrames & 503442.73 & 108047.93 \\
Total & 1086060.82 & 689876.31 \\
\hline
\end{tabular}

el cálculo de los volúmenes por remediar en el sitio.

Las interpolaciones de los dos parámetros (acidez y $\mathrm{pH}$ ), se realizaron con algoritmos diferentes, ya que de esta manera se pudo obtener un mejor ajuste de las interpolaciones a los datos de campo.

La decisión de emplear dos algoritmos de interpolación diferentes para generar los mapas de niveles de contaminación obedeció a las propiedades de los datos a interpolar en relación con su variabilidad y las características de los algoritmos de interpolación. Los datos de acidez tienen un rango de variación grande (de 1.69 a $800.48 \mathrm{cmol} / \mathrm{kg}$ ), mientras que los valores de $\mathrm{pH}$ tienen un rango de variación relativamente corto (de 1.2 a 10.0).

Una de las características del interpolador IDW es que requiere una nube de puntos lo suficientemente densa para mostrar la variabilidad en el terreno (condición alcanzada en este trabajo dado el número de puntos muestreado). Dado que la importancia o peso de un punto en la interpolación es isotrópica, la influencia de un punto en el valor interpolado está en función de las distancias entre éstos. Una de las características del algoritmo IDW es que no genera crestas en las superficies provenientes de la interpolación y dado el rango de valores de los datos de acidez, era necesario evitar este efecto, ya que se habrían obtenido (en la capa resultante) datos que estarían muy por encima o abajo de las mediciones obtenidas en el laboratorio.

El algoritmo de kriging se basa en el supuesto de que cualquier variable que se interpola puede ser considerada como un parámetro regionalizado, es decir que varía en forma continua de un punto a otro. Por tanto, los puntos más cercanos entre sí tienen un cierto grado de correlación espacial y lo puntos más lejanos tienden a ser estadísticamente independientes o no correlacionados (Myers 1991). El kriging ordinario en particular, es un interpolador "lineal" ya que las estimaciones son combinaciones lineales ponderadas de los datos, minimiza el error de la varianza $\mathrm{S}_{\mathrm{R}} \mathrm{Y}_{\mathrm{H}}$ el resultado es el "mejor predictor" (Issaks y Srivastava 1989). Dado que la variación de los datos de $\mathrm{pH}$ es relativamente baja, el algoritmo de kriging produce una superficie muy cercana a los puntos. 
Los valores de $\mathrm{pH}$ obtenidos en el laboratorio oscilan de 1.2 a 10.0 y presentan una variación espacial relativamente suave, por lo que el algoritmo de kriging produjo mejores resultados de interpolación. Los resultados de correlación entre los datos medidos y los datos interpolados es de $\mathrm{R}=0.983$ (Fig. 10).

La plicación de kriging en el programa ArcGIS produce además un archivo que contiene la "predicción de la varianza". Asumiendo que los errores están distribuidos normalmente, hay una probabilidad de $95.5 \%$ de que el valor calculado de z (el parámetro de $\mathrm{pH}$ ) en la celda es el valor predicho, más o menos dos veces la raíz cuadrada del valor en el archivo de predicción resultante. La figura 9 muestra el resultado de este cálculo. Hay que señalar varios puntos respecto de este resultado: a) las desviaciones más altas están en la periferia de la zona, el algoritmo carece de datos en la zona exterior al límite de propiedad y entonces la incertidumbre en la interpolación es mayor, ésto es una ocurrencia común y puede resolverse al obtener puntos de muestreo por afuera de los límites de la zona de estudio. Lo anterior no fue posible dado que el predio estudiado estaba rodeado de terrenos de propiedad privada y no se permitió el acceso a ellos; b) dentro de la zona de estudio la incertidumbre aumenta en aquellas áreas en donde no hay puntos de muestreo, por ejemplo en la laguna que drena la zona de vats (Fig. 9, zona marcada con el número 4) o los sedimentos al norte de la misma laguna (Fig. 9, marcados con el número 1, que no fue posible muestrear ya que este sitio permaneció inundado durante el trabajo de campo) o algunos sitios no contaminados pero con vegetación muy cerrada que impedía el acceso a esas áreas; c) se observa también un efecto de "moteado" ya que el error es comparativamente menor en donde se localizan los puntos empleados para la interpolación.

Los resultados de las estadísticas descriptivas de la correlación para el $\mathrm{pH}$ muestran un valor de la raíz cuadrada del error cuadrático medio (RECM) de 0.253 (Cuadro IV y Fig. 10). Los puntos con una desviación mayor a 1 fueron tres: punto SU35/4, suelo, con una desviación de -1.4; punto SU36/3, suelo, con una desviación de 1.1 y punto SU22/18, suelo, con una desviación de 1.1.

A diferencia del $\mathrm{pH}$, los valores de acidez tienen una variación amplia (de 1.69 a $800 \mathrm{cmol} / \mathrm{kg}$ ), lo que hace que el algoritmo de kriging produzca resultados poco adecuados (muy "suavizados"), suprimiendo los "picos y valles" presentes en los datos puntuales. El algoritmo IDW produce mejores resultados ya que usa una combinación lineal ponderada del conjunto de puntos empleados en el cálculo. La superfi- cie resultante crea una representación más adecuada de los valores obtenidos en el laboratorio, con una correlación de $\mathrm{R}=0.989$ (Cuadro IV y Fig. 11). El valor de RECM de los datos de acidez fue de 11.126, este valor está afectado por los puntos que se desvían de la línea de regresión calculada con una $\mathrm{R}^{2}$ de 0.989 .

\section{CONCLUSIONES}

El uso de datos de percepción remota obtenidos en forma casi simultánea al trabajo de campo facilitó la identificación y localización de muchos rasgos de interés (derrames) en la zona de estudio. Aunado a esto, la disponibilidad de datos de alta resolución espacial permitió localizar derrames de tamaño pequeño, los cuales hubieran pasado desapercibidos en el muestreo de campo dadas las condiciones de vegetación cerrada y alta.

La alta dimensionalidad radiométrica de los datos de imágenes Ikonos (12 bits) fue útil para identificar variaciones en los sitios contaminados, que de otra manera no hubieran podido ser localizados fácilmente.

Estos datos, en combinación con el conocimiento de campo (proporcionado por el personal local) permitieron ubicar más eficientemente los sitios de muestreo para delimitar la extensión lateral de los derrames y poder cuantificar los niveles de contaminación y el cálculo de los volúmenes a remediar. Además, posibilitó el obtener más y mejor información por el mismo costo y visualizar los resultados de manera eficiente.

La combinación de imágenes de alta resolución y el muestreo intensivo, asociado al análisis de laboratorio, permitió generar un mapa adecuado de los niveles de contaminación en la zona de estudio. En dicho mapa se logró mostrar la relación entre los sitios contaminados y otros rasgos de la zona, como caminos, arroyos y lagos, que es información relevante para el diseño de las estrategias de remediación del sitio.

El volumen a remediar dependerá del límite máximo de acidez que establezca la autoridad para ser remediado, ya que se calculó una diferencia de casi $40 \%$ del volumen a remediar con límites de 100 $\mathrm{cmol} / \mathrm{kg}$ vs. $200 \mathrm{cmol} / \mathrm{kg}$.

\section{REFERENCIAS}

Cassard C., Billa M., Lambert A., Picot J., Husson Y., Lasserre L. y Delor C. (2008). Gold predictivity mapping in French Guiana using an expert-guided data-driven 
approach based on a regional-scale GIS. Ore Geol. Rev. 34, 471-500. DOI: 10.1016/j.oregeorev.2008.06.001.

Chica-Olmo M. (1994). Análisis geoestadístico en el estudio de la exploración de recursos minerales. Tesis Doctoral. Universidad de Granada, Granada, España, 387 pp.

Chica-Olmo M. y Luque J.A. (2002) Applications of local estimation of the probability distribution function in environmental sciences by kriging methods. Inverse Probl. 18, 25-36. DOI: 10.1088/0266-5611/18/1/302.

Chica-Olmo M., Rigosol-Sánchez J.P., Pardoigúzquiza E. y García-Soldado M.J. (2008). Análisis espacial de datos geológicos y ambientales: geoestadística, teledetección y SIG. En: Conceptos y técnicas en hidrogeología. Monografías sobre geología aplicada (I. Vadillo, Ed.). Colegio Oficial de Geólogos de Andalucía Ciudad, Andalucía, España, pp. 267-301.

Carranza E. J. M. (2009). Controls on mineral deposit occurrence inferred from analysis of their spatial pattern and spatial association with geological features. Ore Geol. Rev. 35, 383-400. DOI: 10.1016/j.oregeorev.2009.01.001.

García Mora T. y Mas J.F. (2008). Comparación de metodologías para el mapeo de la cobertura y uso del suelo en el sureste de México. Investigaciones Geográficas - Geografía Física 67, 7-19. DOI: http:// dx.doi.org/10.14350/rig. 17987.

Hörig B., Kühn F., Oschütz F. y Lehmann F. (2001). HyMap hyperspectral remote sensing to detect hydrocarbons. Int. J. of Remote Sens. 22, 1413-1422. DOI:10.1080/01431160120909.

Isaaks E.H. y Srivastava R.M. (1989). An introduction to applied geostatistics, Oxford University Press, Nueva York, EUA, 561 pp.

Joan J.V.N. (2005). El papel de la teledetección en el proyecto e-EcoRisk. Memorias. XI Congreso Nacional de Teledetección. Puerto de la Cruz, Tenerife. 21-23 septiembre, pp. 443-446.
Kettles I.M., Rencz A.N. y Bauke D. (2000). Integrating Landsat, geologic, and airborne gamma ray data as an aid to surficial geology mapping and mineral exploration in the Manitouwadge area, Ontario. Photogramm. Eng. Rem. S. 66, 437-445.

Mularz S.C. (1998). Satellite and airborne remote sensing data for monitoring of an open-cast mine. Memorias. Proceedings IAPRS archives, GIS-Between visions and applications, Stuttgart, Alemania. 7-10 septiembre, pp 395-402.

Myers D. E. (1991). Pseudo cross-variograms, positivedefiniteness, and cokriging. Math. Geology 23, 805816. DOI: $10.1007 / \mathrm{BF} 02068776$.

Ponvert-Delisles D. y Lau-Quan A. (2013) Uso de las imágenes de satélites y los SIG en el campo de la Ingeniería Agrícola. Rev. Cie. Téc. Agr. 22, 75-80. [en línea] http://scielo.sld.cu/scielo.php?script=sci arttext\&pid=S2071-00542013000400013\&lng=es\& nrm=iso. ISSN 2071-0054 08/09/2014

Rebordosa X. y López J. (2009). Aplicación de la teledetección en el seguimiento temporal de actividades mineras a cielo abierto. Actualidad Tecnológica Minería, 1-6. [en línea] dialnet.unirioja.es/descarga/ articulo/3395277.pdf 03/09/2014.

Rigol J.P. y Chica-Olmo M. (1998). Merging remotesensing images for geological-environmental mapping: application to The Cabo de Gata-Nfjar Natural Park, Spain. Environ. Geol. 34, 194-202.

Rigol-Sánchez J.P., Chica-Olmo M., Pardo-Igúzquiza E., Rodríguez-Galiano V. y Chica-Rivas M. (2011). Análisis e integración de datos espaciales en investigación de recursos geológicos mediante Sistemas de Información Geográfica. Boletín de la Sociedad Geológica Mexicana 63, 61-70. 